ISSN 0103-5150

Fisioter. Mov., Curitiba, v. 24, n. 3, p. 549-556, jul./set. 2011 Licenciado sob uma Licença Creative Commons

\title{
Efeitos de diferentes programas de exercícios físicos na marcha de idosos saudáveis: uma revisão
}

\author{
Effects of differents schedule of exercise on the gait \\ in healthy elderly: a review
}

\section{Danielle Brandalize ${ }^{[a]}$, Paulo Henrique Foppa de Almeida ${ }^{[b]}$, Juliano Machado ${ }^{[c]}$, Ricelli Endrigo ${ }^{[d]}$, Andressa Chodur ${ }^{[\mathrm{e}]}$, Vera Lúcia Israel ${ }^{[\mathrm{f}]}$}

[a] Fisioterapeuta graduada pela Universidade Estadual do Centro-Oeste (UNICENTRO), Mestre em Comportamento Motor pela Universidade Federal do Paraná (UFPR), Curitiba, PR - Brasil.

[b] Educador físico graduado pela Universidade Federal do Paraná (UFPR), Mestre em Fisiologia da Performance (UFPR), Curitiba, PR - Brasil, e-mail: paulofoppa@hotmail.com

[c] Educador físico graduado pela Universidade da Região de Joinville (UNIVILLE), Mestre em Fisiologia da Performance (UFPR), Curitiba, PR - Brasil, e-mail: jumachado17@yahoo.com.br

[d] Educador físico graduado pela FACEPAL de Palmas, especialista em Fisiologia do Exercício e Atividade Física e Saúde pela Universidade Federal do Paraná (UFPR), Mestre em Fisiologia da Performance (UFPR), Curitiba, PR - Brasil.

[e] Terapeuta ocupacional pela Universidade Federal do Paraná (UFPR), Mestre em Comportamento Motor (UFPR), Curitiba, PR - Brasil, e-mail: andressachodur@hotmail.com

[f] Fisioterapeuta graduada pela Pontifícia Universidade Católica do Paraná (PUCPR), Doutora em Educação Especial pela Universidade Federal de São Carlos (UFSCar), docente do curso de fisioterapia da Universidade Federal do Paraná - setor litoral (UFPR - Litoral), docente do Mestrado em Educação Física da Universidade Federal do Paraná (UFPR), Curitiba, PR Brasil, e-mail: veraisrael@terra.com.br

\section{Resumo}

Introdução: Com o aumento da população idosa e da perda da capacidade funcional com o envelhecimento, há um importante interesse em investigar medidas que possam propiciar um envelhecimento mais saudável. Objetivos: 0 objetivo deste estudo foi, por meio de uma revisão bibliográfica, verificar os efeitos dos diferentes tipos de atividade física na performance funcional da marcha em idosos saudáveis. Materiais e métodos: Para esta revisão a base de dados MEDLINE foi consultada, definiram-se como limites de busca os artigos publicados entre os anos de 1999 e 2008 e que abrangessem o tema atividade física e marcha em idosos saudáveis. Resultados: A maioria dos estudos aponta que programas de exercício estruturados são efetivos em se 
contrapor aos declínios funcionais relacionados com a idade, e os efeitos benéficos do exercício na marcha dos idosos têm sido bem estabelecidos. Conclusão: Os resultados confirmam que o treinamento de força, o treinamento funcional e os exercícios de alongamento têm efeitos positivos em diferentes parâmetros da marcha.

Palavras-chave: Idoso. Marcha. Exercício físico.

\begin{abstract}
Introduction: With the growing of the elderly population and the loss functional capacity with aging there is an important interest to investigate measures that could improving a healthy aging. Objectives: The objective of this study was by a review on the literature searching for the effects of differents types of physical activity in the functional performance of health elderly gait. Materials and methods: To this review search on public data basis in internet was conducted in Medline, between 1999 and 2008, articles that embrace physical activity and health elderly gait were selected. Results: The majority of the studies showed that the exercises programs are effective to put against the progression of frailty age-related and the beneficial effects of exercise in the healthy elderly gait has been well established. Conclusion: In conclusion strength training, funtional exercise and stretching have positive effects to improve the gait performance.
\end{abstract}

Keywords: Elderly. Gait. Exercise.

\section{Introdução}

A Organização Pan-Americana da Saúde (1) define envelhecimento como um processo sequencial, individual, acumulativo, irreversível, universal e não patológico de deterioração de um organismo maduro, próprio de todos os membros de uma espécie, de maneira que o tempo o torne menos capaz de fazer frente ao estresse do meio ambiente e, portanto, aumente sua possibilidade de morte. 0 crescimento da população de idosos, em números absolutos e relativos, é um fenômeno mundial e estudos mostram que é o segmento que cresce mais rapidamente.

Segundo Andrews (2), as projeções indicam que, em 2050, a população idosa será de 1.900 milhões de pessoas, sendo assim necessário criar mecanismos para que os idosos tenham uma condição de vida melhorada em um futuro não muito distante. Atualmente, no Brasil, existem aproximadamente 17,6 milhões de idosos acima de 69 anos, o que corresponde a $8,6 \%$ da população total (3). Estima-se que esse número ultrapasse os 30 milhões em 20 anos (4). Berquó (5) relata que no Brasil, para o ano 2010 , teremos uma cifra de $4,5 \%$ de homens e de $6,76 \%$ de mulheres acima de 65 anos.

A marcha em idosos tem sido investigada desde 1940, com crescente interesse, dada a importância que a manutenção da mobilidade representa para esse grupo de indivíduos, em termos de autonomia e qualidade de vida (6). A velocidade da marcha é um dos aspectos funcionais que mais se altera com o envelhecimento, principalmente após a sétima década de vida (7). Segundo Judge et al. (7), a taxa de declínio da velocidade da marcha é de $12 \%$ a $16 \%$ por década a partir dos 70 anos, já Bohannon (8) reporta que a velocidade da marcha habitual pode diminuir de $9 \%$ a $11 \%$ a partir dos 40 anos. Esse declínio na velocidade da marcha do idoso está relacionado com o aumento no risco de quedas, as quais levam frequentemente à perda da independência $\mathrm{e}$ à institucionalização (7).

Graças a essas perspectivas do aumento da população idosa e da perda da capacidade funcional com o envelhecimento, pesquisadores da área da saúde têm tido grande interesse em pesquisar os benefícios da atividade física e sua influência para um envelhecimento saudável. A participação em atividade física regular, por meio de exercícios aeróbios, de força e de flexibilidade, é uma intervenção efetiva para reduzir ou prevenir declínios funcionais associados ao envelhecimento (9). Os principais benefícios da atividade física regular para idosos são: a redução do risco de doenças cardiovasculares, da hipertensão, da diabetes do tipo 2, da osteoporose, da obesidade, redução do risco de quedas e suas lesões, como também a prevenção de limitações funcionais (10-12).

Portanto, o objetivo desta revisão de literatura é verificar os efeitos do treinamento de força, dos exer- 
cícios funcionais específicos e exercícios de alongamento na marcha de idosos saudáveis.

\section{Metodologia}

Para a elaboração deste estudo, foi realizada uma consulta à base de dados MEDLINE no período de maio a julho de 2008. Definiram-se como limites de busca os artigos, publicados entre os anos de 1999 e 2008, que abrangessem o tema atividade física e marcha de idosos saudáveis. Foram considerados nas buscas os seguintes descritores: "idoso"; "marcha"; "exercício"; "healthy elderly"; "gait"; "exercise".

Os critérios de inclusão adotados foram: artigos publicados na íntegra no período selecionado, estudos experimentais, ensaios controlados e/ou randomizados, em que os resultados apontassem o efeito do exercício sobre a marcha de idosos. Foram excluídos os estudos realizados com idosos que apresentassem alguma patologia e aqueles que avaliaram o efeito do exercício em outra função que não a marcha. Assim, dos 219 estudos listados, 22 foram selecionados por atenderem os critérios impostos.

Alguns estudos clássicos foram mencionados a fim de contextualizar o problema proposto pela pesquisa e também porque fundamentam os conhecimentos sobre exercício físico e marcha.

\section{Revisão de literatura}

É bem estabelecido que os indivíduos que mais se beneficiam do treinamento físico são justamente aqueles que se encontram destreinados. Segundo o Colégio Americano de Medicina e Esporte (ACSM) (13), estudos de curta duração mostraram que a maior parte do ganho de força ocorre nas primeiras quatro a oito semanas de treinamento. A força muscular aumenta aproximadamente $40 \%$ em indivíduos destreinados, $20 \%$ nos moderadamente treinados, $16 \%$ nos treinados, $10 \%$ nos avançados e $2 \%$ em atletas de elite, em períodos de treinamento de força compreendidos entre quatro semanas e dois anos (13).

Nesse sentido, em indivíduos destreinados, pequenos estímulos de treinamento levarão ao desenvolvimento de diversas capacidades físicas, pois o baixo nível de aptidão dessas capacidades é sensível a qualquer dose de trabalho.
É possível perceber um declínio não linear no equilíbrio, força muscular e na marcha com o envelhecimento, o que justifica a necessidade de se preservar os fatores que contribuem para uma maior independência funcional (14). Manter a força dos membros inferiores - uma marcha segura e eficiente - e um bom equilíbrio pode afetar positivamente a saúde e a independência funcional dos idosos, pois quando estes apresentam uma disfunção do equilíbrio, uma pobre marcha e reduzida força muscular aumentam-se os riscos de quedas e fraturas (14).

Em estudo realizado por Manini et al. (15), verificou-se que tanto o treinamento de força como a realização de tarefas funcionais, do tipo subir e descer cadeiras e escadas em vários níveis de dificuldades, ajoelhar-se com uma determinada sobrecarga, levantar e carregar um cesto de roupas até a lavanderia e passar o aspirador de pó, realizadas duas vezes por semana, durante dez semanas, foram efetivos para melhorar a velocidade da marcha em idosos. Dessa forma, mesmo que determinado exercício físico não seja específico para o desenvolvimento da marcha em idosos, ele contribuirá no seu desempenho, pois serão desenvolvidas as capacidades de força, equilíbrio, coordenação, flexibilidade e potência, necessárias à marcha.

No entanto, observando-se o princípio da especificidade do treinamento, para o desenvolvimento da velocidade da marcha, os exercícios específicos de funcionalidade foram superiores ao treinamento geral de força, embora os dois treinamentos tenham sido eficientes (16). Posto isso, nenhum exercício físico parece ser melhor do que a prática da própria marcha para o desenvolvimento da performance funcional da marcha em idosos.

Barrett e Smerdely (17) compararam o treinamento de força com a realização de exercícios de alongamento, onde foi observado que o primeiro foi superior para promover a força, a performance da marcha e o equilíbrio em idosos.

Tem sido demonstrado que a combinação de modelos de exercícios diferentes, como o treinamento de força associado a exercícios aeróbios, ou estes combinados com atividades de equilíbrio, exercícios para coordenação e alongamento, são efetivos para melhorar a performance e a cinemática da marcha (18-20). Diante dos diversos efeitos produzidos pelas diferentes modalidades de exercício físico sobre os parâmetros da marcha, torna-se fundamental analisar os efeitos de cada modalidade isoladamente. 
Importância do treinamento de força na performance da marcha

A perda da massa muscular que acompanha o envelhecimento, fenômeno conhecido como sarcopenia, e o consequente declínio na força muscular, implica significantes consequências relacionadas com a capacidade funcional do idoso (21). Além do mais, o declínio na força muscular é mais acentuado próximo aos 55 anos de idade nas mulheres, e estas apresentam também maior correlação entre força e mobilidade funcional. Já os homens apresentam um decréscimo mais gradual na força e menor correlação entre força e mobilidade funcional (22).

0 treinamento de força tem sido amplamente recomendado para a manutenção da força e função física nos idosos (23). Dessa forma, o maior conhecimento desses efeitos é fundamental para melhor programar políticas de saúde publica ou modelos de tratamentos dessa população, pois se tem mostrado que a prevalência de sarcopenia em idosos saudáveis é de $22,6 \%$ nas mulheres e $26,8 \%$ nos homens (24).

Diversos estudos mostraram resultados positivos com o treinamento de força, na melhora da velocidade (25-27) e estabilidade da marcha em idosos (28). Contudo, no estudo realizado por Sullivan et al. (29), utilizando o treinamento de força e sua influência no desenvolvimento da velocidade da marcha, observou-se uma correlação fraca entre as duas variáveis. Embora os indivíduos idosos que treinaram força melhoraram sua performance funcional da marcha, esse desempenho não foi proporcional aos ganhos de força, indicando a influência de outras variáveis importantes à performance da marcha.

Sayers et al. (30) observaram, em seu experimento, que a velocidade de execução dos movimentos no treinamento de força não influenciou os resultados, já que tanto a alta como a baixa velocidade de execução resultaram em melhorias no equilíbrio dinâmico e na marcha. No entanto, parece existir um limiar ótimo de intensidade para os efeitos do treinamento sobre a marcha em idosos, pois Lopopolo et al. (18) concluíram que o treinamento de força de alta intensidade e alto volume foi mais eficiente para melhorar a velocidade da marcha quando comparada ao treinamento de força com intensidade baixa e moderada ou volume baixo.

Como mencionado anteriormente, vários mecanismos são responsáveis pela melhora da performance da marcha nos idosos que realizam exercícios físicos.
No entanto, os benefícios não estão inteiramente relacionados com o treinamento físico, pois a nutrição também contribui de forma positiva para os ganhos. Em um estudo prospectivo com idosos deficientes de vitamina $D$, verificou-se que, com apenas o treinamento de força, ocorreram significativas melhoras na força muscular e na velocidade da marcha. Contudo, indivíduos que além do treino de força foram suplementados com vitamina $\mathrm{D}$ e cálcio mostraram ganhos superiores (31). Uma explicação plausível para esses efeitos é o fato de o metabólito ativo da vitamina D se ligar com alta especificidade no seu receptor nuclear no tecido muscular, levando a um aumento da força e da massa muscular, contribuindo para reduzir os riscos de quedas nessa população (32).

Diante de todas as citações acima, é possível verificar que os idosos são altamente adaptáveis ao treinamento de força, e esse é um fator muito positivo para se contrapor aos efeitos deletérios que acompanham a idade. Entretanto, essa plasticidade muscular ocorre nos dois sentidos, isto é, com o destreinamento após um programa combinado de treinamento de força e condicionamento aeróbio, os ganhos na velocidade da marcha, subida e descida de escadas retornaram aos valores pré-treinamento após 1, 2 e 3 meses da interrupção desse programa, respectivamente (33). Embora neste estudo os ganhos na qualidade de vida não se alteraram com o destreinamento, os autores da pesquisa concluíram que os programas de exercícios com idosos não deveriam ser interrompidos, para que sejam mantidos os benefícios adquiridos.

\section{Importância dos exercícios funcionais específicos da marcha}

Exercícios funcionais de alta intensidade são efetivos para a melhora da performance da marcha (34). Quando comparado o treinamento específico para a marcha com o treino de equilíbrio estático, o primeiro foi superior para o desenvolvimento da velocidade da marcha e do equilíbrio dinâmico, sendo ambos os treinamentos eficientes para o desenvolvimento do equilíbrio estático (35).

Em estudo com idosos hospitalizados, os quais foram randomicamente divididos em grupo controle e intervenção e submetidos a sessões de treinamento, foi verificada melhora da performance da marcha em idosos que realizaram a combinação de 
movimentos de tai chi chuan e atividades funcionais, como transferência entre cadeiras, deslocamento de peso, alcançar e caminhar (36).

Dada a importância do exercício específico de caminhada para o desenvolvimento e o desempenho dessa atividade, Marsh et al. (37) compararam dois programas de caminhada, em esteira e no solo, para verificar possíveis diferenças nos resultados dessas duas atividades aparentemente similares. A conclusão foi de que o programa de caminhada no solo foi superior ao programa de caminhada na esteira na performance da marcha dos idosos. Vale ainda citar que a própria atividade de caminhada está positivamente correlacionada à qualidade óssea entre as idosas (38).

Importância dos exercícios de alongamento na performance da marcha

A flexibilidade é um termo geral que abrange a amplitude de movimento de uma ou múltiplas articulações, com a amplitude de movimento dependente dos tecidos ósseos, musculares e do conectivo (9). A idade por sua vez afeta a estrutura desses tecidos, tanto que a função em termos de flexibilidade específica e amplitude de movimento nas articulações, para o desempenho de tarefas motoras grossas, é reduzida (9).

Há tempos, não se sabia se os parâmetros biomecânicos da marcha de idosos estavam relacionados com a idade por si só ou se estavam relacionados com uma reduzida velocidade de caminhada (39). Nesse sentido, Kerrigan et al. (39) objetivaram identificar as mudanças biomecânicas específicas, independente da velocidade, que pudessem prejudicar a performance da marcha em idosos saudáveis e determinar se essas mudanças persistiam com o aumento da velocidade de caminhada. Foi verificado que a performance da marcha em idosos pode ser limitada tanto por uma sutil contratura dos flexores do quadril como por uma fraqueza concêntrica dos músculos flexores plantares do tornozelo. Posteriormente, foi mostrado que a redução relacionada com a idade no pico de extensão do quadril durante a marcha era resultado de uma contratura do quadril estática ao invés de uma consequência dinâmica (40).

De acordo com Lee et al. (41), em velocidade confortável de caminhada os idosos apresentavam significativa redução no pico de extensão de quadril ( $7 \pm 6$ graus vs. $11 \pm 6$ graus) e báscula do quadril aumentada ( $15 \pm 5$ graus vs. $11 \pm 5$ graus) em comparação aos jovens adultos. Além do mais, na caminhada em velocidade rápida o pico de extensão do quadril é significativamente reduzido nos idosos em comparação aos adultos jovens $(9 \pm 6$ graus vs. $14 \pm 6$ graus). Entretanto, não houve diferença significativa na velocidade lenta em ambos os grupos, mostrando novamente que as alterações na marcha, relacionadas com a idade, são definidas por um pico reduzido de extensão do quadril e báscula do quadril aumentada, que são características posturais dinâmicas ao invés de características posturais estáticas.

Em um programa de treinamento de flexibilidade domiciliar para os músculos flexores do quadril, realizado duas vezes por dia durante dez semanas, ocorreu uma modesta melhora no pico de extensão estática do quadril em comparação ao grupo controle, além de que as mudanças na cinemática e cinética da marcha incluíram significativa melhora nos músculos flexores plantares do tornozelo do grupo tratamento em comparação ao grupo controle (40).

No estudo de Rodacki et al. (42), no qual foi realizada uma única sessão de exercício de alongamento estático para os músculos flexores e extensores do quadril, observou-se uma velocidade maior de marcha, maior comprimento dos passos e menor tempo de sustentação dupla dos pés durante a posição ortostática. Tais resultados sugerem maior estabilidade e mobilidade com a realização de exercícios de alongamento, mostrando que essa é uma excelente modalidade terapêutica e promissora em facilitar as mudanças dos parâmetros da marcha, contribuindo também para a redução dos riscos de quedas (42).

Esses resultados mostram que os idosos, ao iniciarem um programa de exercícios de alongamento visando a melhorar a performance da marcha, obterão efeitos benéficos tanto com uma sessão única de exercícios como com um programa mais duradouro. No entanto, não se deve esquecer que os ganhos são perdidos logo após a descontinuidade da prática regular de exercícios físicos.

\section{Considerações finais}

Com base em resultados encontrados, pode-se dizer que há muito existe um consenso dos pesquisadores sobre a relação positiva entre exercício físico e performance da marcha em idosos saudáveis, e esse consenso se mantém atual. Dessa maneira, é possível 
afirmar que a diminuição que ocorre com o passar dos anos de certas capacidades, essenciais para uma boa performance da marcha como força, equilíbrio, coordenação, flexibilidade e potência, pode ser compensada em diferentes graus com o exercício físico.

Viu-se que estudos que relacionaram a marcha a treinamento de força, treinamento funcional e exercícios de alongamento encontraram resultados positivos em diferentes parâmetros da marcha. No entanto, ao se interromper o programa de exercícios, os ganhos são perdidos. Com isso, é importante que, com o planejamento dos exercícios, se fizesse um planejamento para a aderência dos idosos aos programas, já que os exercícios, tanto de treinamento de força quanto de alongamento e funcionais, melhoram o desempenho da marcha de idosos saudáveis, e essa é uma habilidade essencial para a realização das atividades da vida diária e independência.

Deve-se levar em consideração que esta revisão limitou-se em utilizar artigos encontrados em um único banco de dados, fazendo-se necessários novos estudos que incluam um maior número de base de dados em suas buscas por evidências científicas.

\section{Referências}

1. Organización Panamericana de la Salud. Guía clínica para atención primaria a las personas mayores. Washington: OPAS; 2003.

2. Andrews GA. Los desafíos del proceso de envejecimiento en las sociedades de hoy y del futuro. Encuentro Latinoamericano y Caribeño sobre las Personas de Edad. Santiago: Celade; 1999. p. 274-56.

3. Instituto Brasileiro de Geografia e Estatística - IBGE. Síntese de indicadores sociais. Estudos e pesquisas. Informação demográfica e socioeconômica. Rio de Janeiro: IBGE; 2000. n. 4, p. 226.

4. Instituto Brasileiro de Geografia e Estatística - IBGE. Síntese de indicadores sociais. Estudos e pesquisas. Informação demográfica e socioeconômica. Rio de Janeiro: IBGE; 2004. n. 5, p. 369.

5. Berquó E. Algumas considerações demográficas sobre o envelhecimento da população no Brasil. In: Anais do I Seminário Internacional do Envelhecimento Populacional: uma agenda para o final do século. Brasília: Ministério da Previdência e Assistência Social, Secretaria da Assistência Social, 1996. p. 16-34.
6. Castro CLN, Santos JACB, Leifeld PS, Bizzo LV, Silva LC, Almeida TF, et al. Estudo da marcha em idosos - resultados preliminares. Acta Fisiátrica. 2000;3:103-7.

7. Judge JO, Ounpuu S, Davis RB III. Effects of age on the biomechanics and physiology of gait. Clin Geriatr Med. 1996;12(4):659-78.

8. Bohannon RW. Comfortable and maximum walking speed of adults aged 20-79 years: reference values and determinants. Age Ageing. 1997;26(1):15-9.

9. Pollock ML, Gaesser GA, Butcher JD, Després J, Dishman RK, Franklin, et al. The recommended quantity and quality of exercise for developing and maintaining cardiorrespiratory and muscular fitness, and flexibility in healthy adults. Med Sci Sports Exerc. 1998;30(6):975-91.

10. American Geriatrics Society, British Geriatrics Society, American Academy of Orthopaedic Surgeons Panel on Falls Prevention. Guideline for the prevention of falls in older persons. J Am Geriatr Soc. 2001;49(5): 664-72.

11. Kesaniemi YE, Danforth EJ, Jensen M, Kopelman P, Lefebvre P, Reeder B. Dose-response issues concerning physical activity and health: an evidence-based symposium. Med Sci Sports Exerc. 2001;33(6 Suppl): 351-8.

12. Keysor J. Does late-life physical activity or exercise prevent or minimize disablement? A critical review of the scientific evidence. Am J Prev Med. 2003;25 (3 Suppl 2):129-36.

13. Kraemer WJ, Adams K, Cafarelli E, Dudley GA, Dooly C, Feigenbaum MS, et al. American college of sports medicine. Position stand: progression models in resistance training for healthy adults. Med Sci Sports Exerc. 2002;34(2):364-80.

14. Haber EL, Erbas B, Hill KD, Wark JD. Relationship between age and measures of balance, strength and gait: linear and non-linear analyses. Clin Sci. 2008; 114(12):719-27.

15. Manini T, Moshe M, VanAman T, Cook S, Femhall B, Burke J, et al. Efficacy of resistance and task-specific exercise in older adults who modify tasks of everyday life. J Gerontol A Biol Sci Med Sci. 2007;62(6):16-23.

16. Krebs DE, Scarborough DM, McGibbon CA. Functional vs. strength training in disabled elderly outpatients. Am J Phys Med Rehabil. 2007;86(2):93-103. 
17. Barret CJ, Smerdely P. A comparison of communitybased resistance exercise and flexibility exercise for seniors. Aust J Physiother. 2002;48(3):215-9.

18. Lopopolo RB, Greco M, Sullivan D, Craik RL, Mangione KK. Effect of therapeutic exercise on gait speed in community-dwelling elderly people: a meta-analysis. Phys Ther. 2006;86(4):520-40.

19. Rubenstein LZ, Josephson KR, Trueblood PR, Loy S, Harker JO, Pietruszka FM, et al. Effects of a group exercise program on strength, mobility, and falls among fall-prone elderly men. J Gerontol A Biol Sci Med Sci. 2000;55(6):M317-21.

20. Cao BZ, Maeda A, Shima N, Kurata H, Nishizono H. The effect of a 12-week combined exercise intervention program on physical performance and gait kinematics in community-dwelling elderly women. J Physiol Anthropol. 2007;26(3):325-32.

21. Mazzeo RS, Cavanagh P, Evans WJ, Fiatarone M, Hagberg J, Mcauley E, et al. ACSM position stand on exercise and physical activity for older adults. Med Sci Sports Exerc. 1998;30:992-1008.

22. Samson M, Meeuwsen IBAE, Crowe A, Dessens JAG, Duursma AS, Verhaar HJJ. Relationship between physical performance measures, age, height and body weight in healthy adults. Age Ageing. 2000;29(3):235-42.

23. Misic MM, Rosengren KS, Woods JA, Evans EM. Muscle quality, aerobic fitness and fat mass predict lowerextremity physical function in community-dwelling older adults. Gerontology. 2007;53(5):260-6.

24. Iannuzzi-Sucich M, Prestwood KM, Kenny AM. Prevalence of sarcopenia and predictors of skeletal muscle mass in healthy, older men and women. J Gerontol A Biol Sci Med Sci. 2002;57(12):M772-7.

25. Hauer K, Rost B, Rütschle K, Opitz H, Specht N, Bärtsch P, et al. Exercise training for rehabilitation and secondary prevention of falls in geriatric patients with a history of injurious falls. J Am Geriatr Soc. 2001;49(1):10-20.

26. McCool JF, Schneider JK. Home-based leg strengthening for older adults initiated through private practice. Prev Med. 1999;28(2):105-10.

27. Jette AM, Lachman M, Giorgetti MM, Assmann SF, Harris BA, Levenson C, et al. Exercise-it's never too late: the strong-for-life program. Am J Public Health. 1999;89(1):66-72.
28. Hausdorff JM, Nelson ME, Kaliton D, Layne JE, Bernstein MJ, Nuernberger A, et al. Etiology and modification of gait instability in older adults: a randomized controlled trial of exercise. J Appl Physiol. 2001;90(6):2117-29.

29. Sullivan DH, Wall PT, Bariola JR, Bopp MM, Frost YM. Progressive resistance muscle strength training of hospitalized frail elderly. Am J Phys Med Rehabil. 2001;80(7):503-9.

30. Sayers SP, Bean J, Cuoco A, LeBrasseur NK, Jette A, Fielding RA. Changes in function and disability after resistance training: does velocity matter?: a pilot study. Am J Phys Med Rehabil. 2003;82(8):605-13.

31. Bunout D, Barrera G, Leiva L, Gattas V, de la Maza MP, Avendaño M, et al. Effects of vitamin D supplementation and exercise training on physical performance in Chilean vitamin D deficient elderly subjects. Exp Gerontol. 2006;41(8):746-52.

32. Bischoff-Ferrari HA. Vitamin D and muscle function Int Congr 2007;1297:143-47.

33. Teixeira-Salmela LF, Santiago L, Lima RC, Lana DM, Camargos FF, Cassiano JG. Functional performance and quality of life related to training and detraining of community-dwelling elderly. Disabil Rehabil. 2005; 27(17):1007-12.

34. Rosendahl E, Lindelöf N, Littbrand H, Yifter-Lindgren E, Lundin-Olsson L, Haglin L, et al. High-intensity functional exercise program and protein-enriched energy supplement for older persons dependent in activities of daily living: a randomised controlled Trial. Aust J Physiother. 2006;52(2):105-13.

35. Shimada H, Uchiyama Y, Kakurai S. Specific effects of balance and gait exercises on physical function among the frail elderly. Clin Rehabil. 2003;17(5): 472-9.

36. Haines TP, Hill KD, Bennell KL, Osborne RH. Additional exercise for older subacute hospital inpatients to prevent falls: benefits and barriers to implementation and evaluation. Clin Rehabil. 2007;21(8): 742-53.

37. Marsh AP, Katula JA, Pacchia CF, Johnson LC, Koury KL, Rejesky WJ. Effect of treadmill and overground walking on function and attitudes in older adults. Med Sci Sports Exerc. 2006;38(6):1157-64. 
38. Yanagimoto Y, Oshida Y, Sato Y. Effects of walking on bone quality as determined by ultrasound in the elderly. Scand J Med Sci Sports. 2000;10(2):103-8.

39. Kerrigan D, Todd M, Della Croce U, Lipsitz L, Collins J. Biomechanical gait alterations independent of speed in the healthy elderly: evidence for specific limiting impairments. Arch Phys Med Rehabil.1998;79(3): 317-22.

40. Kerrigan DC, Xenopoulos-Oddsson A, Sullivan MJ, Lelas JJ, Riley PO. Effect of a hip flexor-stretching program on gait in the elderly. Arch Phys Med Rehabil. 2003;84(1):1-6.

41. Lee LW, Zavarei K, Evans J, Lelas JJ, Riley PO, Kerrigan DC. Reduced hip extension in the elderly: dynamic or postural? Arch Phys Med Rehabil. 2005;86(9): 1851-4.
42. Rodacki ALF, Souza RM, Ugrinowitsch C, Cristopolisk F, Fowler NE. Transient effects of stretching exercises on gait parameters of elderly woman. Man Ther. 2009; 14(2):167-72.

Recebido: 20/08/2010 Received: 08/20/2010

Aprovado: 01/04/2011

Approved: 04/01/2011 